\title{
New Line Narrowing Effects in the Infrared Collision-Induced Spectra of Molecular Hydrogens in Liquid Neon
}

\author{
W. A. Herrebout, ${ }^{1}$ B. J. van der Veken, ${ }^{1}$ and A. P. Kouzov ${ }^{2, *}$ \\ ${ }^{1}$ Department of Chemistry, University of Antwerp, Antwerp, Belgium \\ ${ }^{2}$ Institute of Physics, Saint Petersburg State University, Peterhof, Saint Petersburg 198504, Russia
} (Received 19 October 2006; published 26 August 2008)

\begin{abstract}
The first spectroscopic observation of the relative (solute-solute) diffusion in a fluid environment is reported. New, unusually sharp $Q_{1}^{q}(J)$ lines developing against the diffuse background in the collisioninduced fundamental IR bands of hydrogen isotopomers $\left(\mathrm{H}_{2}, \mathrm{D}_{2}\right.$, and $\left.\mathrm{HD}\right)$ dissolved in liquid $\mathrm{Ne}(T \approx$ $25 \mathrm{~K})$ are studied as functions of the solute concentration $x$. In all cases, the $Q_{1}^{q}$ intensity parabolically scales with $x$, accompanied by a striking narrowing of the line shapes. The narrowing, as revealed by the $p-\mathrm{H}_{2}$ solution studies, is due to a faster growth with $x$ of the sharper solute-solute induced component of the single $Q_{1}^{q}(0)$ line. The latter as well as other observed solute-solute lines are strongly narrowed by fast velocity decorrelations and are signatures of microscopic-scale diffusion. Also, a first observation of the solute-solvent induced $J \rightarrow J+4$ transitions is reported.
\end{abstract}

PACS numbers: 33.20.Ea, 34.90.+q, 61.25.Bi

In contrast to allowed optical spectra, the collisioninduced absorption (CIA) spectra stem from the polarization $\mathbf{M}$ depending on the particle relative positions and, thereby, provide direct signatures of molecular translations in fluids. For instance, the fast rattling motion of a light solute (guest) trapped inside its first coordination sphere splits the pure vibrational $\Delta J=0$ branch $Q_{1}$ ( $J$ is the rotational quantum number) into $Q_{1}^{p}-Q_{1}^{r}$ doublet, as was observed in the fundamental CIA bands of molecular hydrogens in liquefied noble gases [1]. However, manifestations of the much slower diffusional processes in the CIA spectra so far remain questionable. The diffusion model [2] used to explain the buffer-gas narrowing of the $S_{1}(J)$ lines with $\Delta J=2$ in the $\mathrm{H}_{2} / \mathrm{D}_{2}$ fundamentals [3] was later found to disagree with the newer observations [4] at fluid densities. In our opinion, the revealed inconsistencies are due to the many-body (solute and near solvent particles) nature of $\mathbf{M}$, for its evolution can be hardly described in terms of the solute diffusion only. Instead, we will concentrate on transitions for which $\mathbf{M}(\mathbf{R})$ characterizes a particular pair with separation $\mathbf{R}$. This is the case when (a) two guests meet in the bulk of solvent so that one or both can be optically excited (single or double transitions), and when (b) the guest interacts with a long-living cell defect (a vacancy). In both cases, sharp Lorentzians, quite untypical for the CIA spectra of fluids, arise. As finally concluded, the shape form suggests $\mathbf{R}(t)$ to be converted by fast velocity decorrelations into slow, diffusionlike motion [5]. Notice that at small solute mole fractions $x$, the (a) and (b) spectral signatures scale as $x^{2}$ and $x$, respectively, and can be thus separated.

This study was stimulated by the recently observed [6] unusual behavior of the sharp $Q_{1}^{q}(0)$ line of HD in liquid neon: by increasing $x$ just from 0.01 to 0.04 , the line width [half width at half height (HWHH)] dropped by one-third (from 3.2 to $2.1 \mathrm{~cm}^{-1}$ ). The line sharpness and the abnor- mally strong narrowing (instead of conventional concentration broadening) posed a paradox which at that moment could not be resolved because of the lack of experimental data. To unravel the situation, extensive concentration studies of the fundamental CIA bands of all stable hydrogen isotopomers have now been performed at a higher experimental level. Thanks to a refined shape model, the intense background absorption is fitted to within the noise level, allowing accurate separation of the resonance profiles. Their analysis clearly shows how the sharper guestguest component progressively predominates over the guest-surrounding contribution, resulting in the parabolic intensity increase with $x$ and overall profile narrowing. The use of neon, the coolest feasible solvent $(T \approx 25-40 \mathrm{~K}$, $x \lesssim 0.06)$ perfectly transparent in IR, and molecular hydrogens, manifesting almost unhindered quantized rotation, minimizes the line overlap and facilitates the shape analysis.

Experimental.-The following cryosolutions in liquid Ne were studied: (I) $\mathrm{H}_{2}(T=25.7 \mathrm{~K})$ with nonequilibrium orthopara ratios $\left(\chi_{o / p}=1.7-2.2\right)$, (II) paraenriched $\mathrm{H}_{2}$ $\left(\chi_{o / p} \leq 0.03\right)$ at $T=25.0 \mathrm{~K}$, (III) $\mathrm{HD}$ at $T=25.0 \mathrm{~K}$, (IV) $\mathrm{D}_{2}$ at $T=25.7 \mathrm{~K}$. For systems I-III, $x$ was gradually decreased from 0.05 to 0.003 so that $15-25$ spectra were measured for each system. The premixed-gas concentration uncertainties were less than $3 \%$. The $\mathrm{D}_{2}$ spectra were found to be less accurate because of their overlap with that of traces of water, but the $Q_{1}^{q}$ concentration narrowing was nevertheless apparent. The fundamental spectra were recorded at $0.5 \mathrm{~cm}^{-1}$ resolution with a Bruker IFS $66 \mathrm{v}$ interferometer, a $\mathrm{Si} / \mathrm{CaF}_{2}$ beam splitter, a tungsten source, and an InSb detector, by averaging 1000 scans and applying Blackmann Harris apodization. Gas samples of HD and $\mathrm{D}_{2}$ (Isotec, 98\%), $\mathrm{H}_{2}$ (Air Liquide, N60), and $\mathrm{Ne}$ (Air Liquide, N45) were used without further purification. The sample cells, with path length of 4.0 and $6.0 \mathrm{~cm}$, and 
equipped with quartz or sapphire windows, were attached to the cold head of a Leybold ROK 10-300 double stage cryostat. The temperature was measured with two Si diodes located at the top and at the bottom of the cell bodies. Both cells were equipped with a small compartment designed to host hydrated iron(III)oxide, which catalyzes the $o-p$ conversion. For the spectra of $n-\mathrm{H}_{2}, \mathrm{HD}$, and $\mathrm{D}_{2}$, premixed gases were condensed in the sample cell in the absence of the catalyst. In the $p-\mathrm{H}_{2}$ case, the required amount of $\mathrm{H}_{2}$ was condensed onto the catalyst in the sample cell, at $18 \mathrm{~K}$, and stored for $60 \mathrm{~min}$. Subsequently, the cell was allowed to warm up to $25 \mathrm{~K}$ and solvent gas, precooled with $\mathrm{LN}_{2}$, was slowly added. During condensation of the solvent, the temperature of the solution did not exceed $28 \mathrm{~K}$. The use of a longer path length, as well as of quartz or sapphire windows instead of silicon ones, considerably increased the signal-to-noise ratio, so that weaker features could be studied with a much higher accuracy than previously [6].

Experimental results and their fitting.-In series I, a partially resolved doublet of the $Q_{1}^{q}(0)$ and $Q_{1}^{q}(1)$ lines (data not shown) appears near $\nu_{0}\left(\mathrm{H}_{2}\right)=4161.13 \mathrm{~cm}^{-1}$. Its evolution with $x$ reveals three remarkable effects: (A) The doublet becomes better resolved, implying line narrowing, (B) the integrated intensity grows parabolically, with a nonzero linear term, (C) both lines are simultaneously shifted to the red. Although these facts suggest the guest-guest interactions to play a significant role, a resolution of the apparent paradox (A) demands a derivation of individual line shapes which, in view of their strong overlap, is problematic. Besides, the quadrupole fields of $o$ molecules affect the intensities of both lines and mask the role of a possible isotropic, i.e., rotation-independent, induction mechanism. Moreover, the overall spectral fittings show $\chi_{o / p}$ to slowly drift from 2.2 to 1.7 as the solution was diluted, which can affect the doublet intensity. To simplify the analysis, the $Q_{1}^{q}(1)$ line should be damped, as was practically achieved in series II and III.

The single and double $p-\mathrm{H}_{2}$ transitions are easily distinguished by comparing the spectra detected at different $x$ (Fig. 1). Weak $U_{1}(J)(J=0,1)$ transitions with $\Delta J=4$ are resolved in the $o / p$ nonequilibrium solution of hydrogen. The inset of Fig. 1 shows the $U_{1}(0)$ feature in the $p-\mathrm{H}_{2}$ solution. The sharper $U_{1}^{q}(J)$ components scale linearly with $x$ proving their guest-host induction which, to our knowledge, has never been reported for these transitions. Besides, the apparent $U_{1}^{q}-U_{1}^{r}$ splitting is the same as the $Q_{1}^{q}-Q_{1}^{r}$ and $S_{1}^{q}-S_{1}^{r}$ ones suggesting all of them to be caused by intracell oscillations.

The singlet $Q_{1}^{q}(0)$ resonance line in series II develops in the center of the strongly asymmetric diffuse $Q_{1}^{p}-Q_{1}^{r}$ doublet (see Fig. 1) which dominates the low frequency part of the spectrum and scales linearly with $x$. To study the concentration effects on the $Q_{1}^{q}(0)$ line, especially at very small $x$, one needs to accurately separate the background

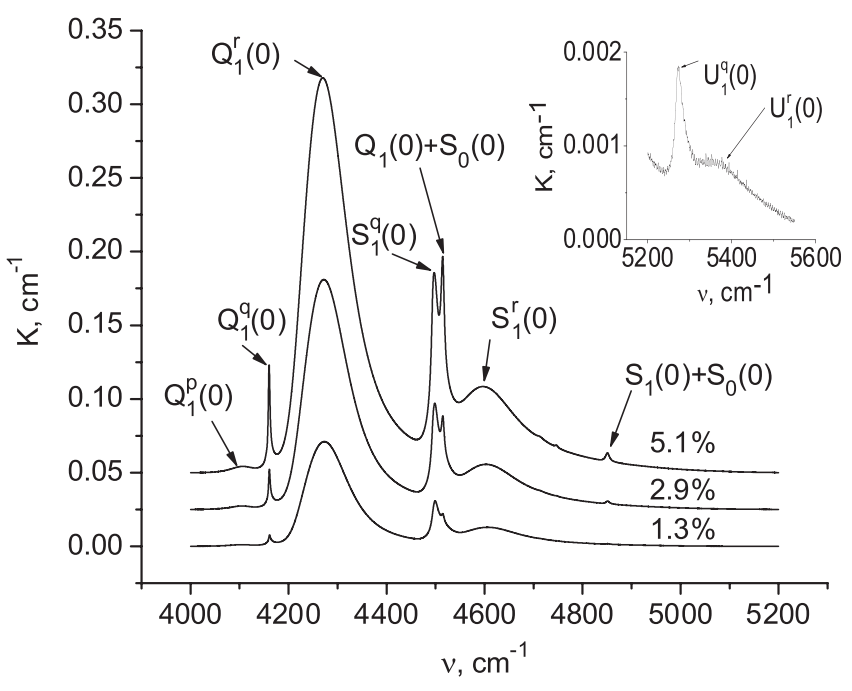

FIG. 1. Absorption coefficient $K(\nu)$ of $p-\mathrm{H}_{2}$ solution in liquid neon $(T=25.0 \mathrm{~K})$ at different concentrations (mol \%). Curves are shifted by 0.025 . Inset shows the spectrum near the $U_{1}(0)$ transition.

intensity. In our previous study of HD in LNe this problem was principally resolved on the basis of the cell model. However, the increased accuracy of new spectra demands finer fittings, and this is also crucial for quantitative studies of other vibrotational lines developing on the background. To that aim, the asymmetric $Q_{1}^{p}-Q_{1}^{r}$ doublet shape $A(z)$ ( $z$ is the detuning from the band origin) is expressed as a sum of the terms corresponding to excitation and deexcitation of the rattling mode at the frequency $\omega_{r}: A(z)=$ $W_{0}\left(z-\omega_{r}\right)+\exp \left(-\hbar \omega_{r} / k T\right) W_{0}\left(z+\omega_{r}\right)$. A refined form of the intracollisional spectrum $W_{0}(u)$ [6] produced by impacts of a guest with the nearest atoms now incorporates the Egelstaff quantum asymmetry factor [7] and the slower decay $\exp \left[-\left(\pi u / 2 \omega_{c}\right)^{2 / 3}\right][8]$ at detunings $u$ much greater than the inverse collision duration $\omega_{c}$. The new fitting function requires one additional parameter and will be described in a separate paper. In the range $\omega / 2 \pi c \leq$ $4450 \mathrm{~cm}^{-1}$ free from $S$ lines the optimized profiles reproduce the measured intensity to within the noise level. Besides, the $4130-4190 \mathrm{~cm}^{-1}$ interval encompassing the $Q_{1}^{q}(0)$ line was excluded from fitting. The resonance shapes were then deduced as the difference between the measured and simulated profiles. These shapes (Fig. 2) are strongly dependent on $x$ and exhibit a clear concentration narrowing. At small $x \leqq 0.01$, the integrated intensity (zero moment) $M_{0}$ varies linearly, $M_{0}=A_{1} x$ with $A_{1}=6.7(2) \mathrm{cm}^{-2}$. Given this value, the total set of measurements is well fitted by the parabola $M_{0}=A_{1} x+A_{2} x^{2}$ (see inset of Fig. 2) with $A_{2}=90(2) \mathrm{cm}^{-2}$. The line shift $\Delta \nu_{m}$ relative to the gas phase frequency scales linearly: $\Delta \nu_{m}(x)=0.23(2)-$ 21(1) $x$. In $\mathrm{cm}^{-1}$ /amagat units, the solute-solute shift coefficient $(-0.015)$ is close to the value extrapolated from the hydrogen gas data [9]. 


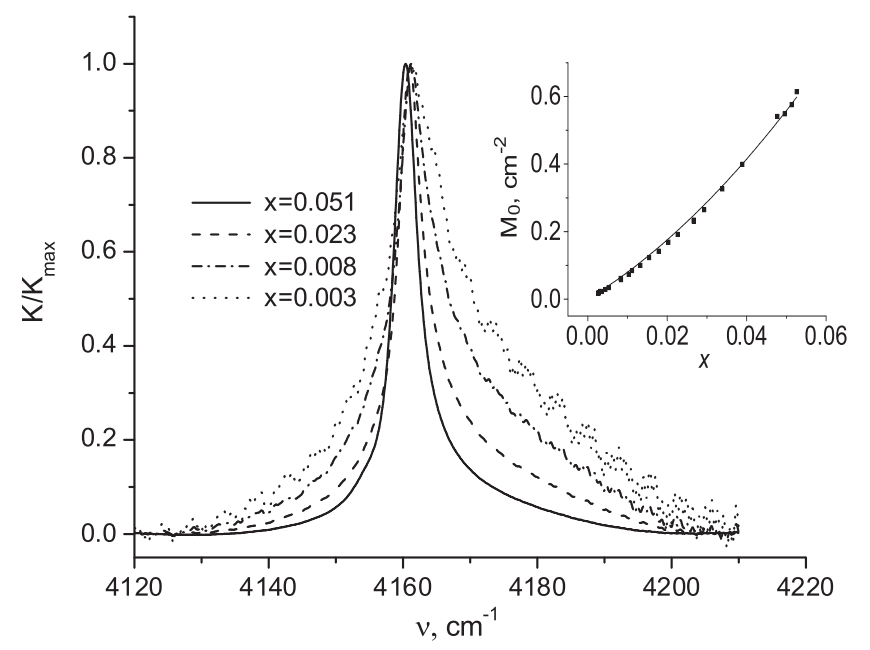

FIG. 2. Normalized $Q_{1}^{q}(0)$ shapes of $p-\mathrm{H}_{2}$ solution $(T=$ $25.0 \mathrm{~K})$ at different concentrations $x$. Integrated $Q_{1}^{q}(0)$ absorption coefficient $\mathrm{M}_{0}$ vs $x$ is plotted in inset: squares-experimental $\mathrm{M}_{0}$ values; solid line-parabolic fit.

For HD, the $Q_{1}^{q}(0)$ shape behaves similarly with $x$ : $\Delta \nu_{m}(x)=0.3(1)-16(2) x ; M_{0}=6.5(5) x+80(12) x^{2}$. In highly diluted nonequilibrium hydrogen solutions $(x \leq$ $0.005)$, the integrated intensity of the $Q_{1}^{q}$ doublet scales linearly with $A_{1}=7.6(5) \mathrm{cm}^{-2}$, which practically coincides with the $p-\mathrm{H}_{2}$ value. In the $p-\mathrm{H}_{2}$ solution, the single $Q_{1}(0)$ line is allowed only for the isotropic induction. The latter induces the $Q(J)$ transitions whose total intensity is not subjected to $\chi_{o / p}$. The coincidence of the $A_{1}$ values for both $\mathrm{H}_{2}$ solutions thus shows the isotropic induction to dominate also in the $o-\mathrm{H}_{2}$ interactions with neon. However, the binary coefficient of the $Q_{1}^{q}(0)-Q_{1}^{q}(1)$ doublet $\left[A_{2} \approx\right.$ $465(15) \mathrm{cm}^{-2}$ ] is 5 times larger than that of the $p-\mathrm{H}_{2}$ solution. The enhancement certainly owes to the anisotropic polarization $\mathbf{M}^{(\mathrm{an})}$ produced by collisions of quadrupolar $o$ molecules with $o$ and $p$ solutes that activates both $Q_{1}^{q}(0)$ and $Q_{1}^{q}(1)$ transitions. The increase of $A_{2}$ thus suggests the quadrupole-induced guest-guest polarization $\mathbf{M}^{(\mathrm{an})}$ to be stronger than the isotropic one, in contrast to what was concluded for the guest-host case. At least partly, this can be explained by the twofold polarizability increase when $\mathrm{Ne}$ is substituted by $\mathrm{H}_{2}$.

Collision-induced polarization in solutions.-To a good approximation, $\mathbf{M}$ is a sum of the binary guest-guest $\left(\mathbf{m}_{a b}\right)$ and guest-host $\left(\mathbf{d}_{c \alpha}\right)$ dipole moments. For a diluted mixture of $N_{1}$ solute and $N$ solvent particles [ $x=N_{1} /\left(N_{1}+N\right) \ll$ 1], the total spectrum can be written as the Fourier transform of $C(t) \approx N_{1}\left\langle\left(\mathbf{M}_{c}(0), \mathbf{M}_{c}(t)\right)\right\rangle+N_{1}^{2}\left\langle\left(\mathbf{m}^{\prime}(0), \mathbf{m}^{\prime}(t)\right)\right\rangle$ where $\mathbf{M}_{c}=\sum_{\alpha} \mathbf{d}_{c \alpha}$ is induced by an isolated guest $c$ immersed in a pure solvent. Since the medium is polarizable, the effective guest-guest polarization $\mathbf{m}^{\prime}$ may differ from $\mathbf{m}_{a b}$. However, if one disregards the inhomogeneity of the local surrounding, $\mathbf{m}^{\prime}$ should depend on the same separation $\mathbf{R}_{a b}$ as $\mathbf{m}_{a b}$ and, consequently, its spectrum can be motionally narrowed. Also, because of the large mass difference, the guest moves much faster than the surrounding atoms so that their configuration $(\mathbf{Q})$ may be assumed to be frozen. The observed response is then an average over all possible $\mathbf{Q}$. When the cell is almost spherically symmetric, $\mathbf{M}_{c}(\mathbf{Q})$ behaves as a collective quantity and gives rise to the background absorption whose shape resembles the damped-oscillation features of the solute velocity spectrum. Conversely, when a strong local defect (vacancy) emerges, $\mathbf{M}_{c}$ acquires a component $\mathbf{M}_{c}^{(\mathrm{vac})}(\mathbf{R})$ which is primarily modulated by the vacancy-solute distance $\mathbf{R}$ and evolves in time similarly to $\mathbf{m}^{\prime}$, providing another access to relative diffusion (see below). Actually, a substantial density decrease at melting (for $\mathrm{Ne}$ by about $15 \%$ ) implies almost each cell to contain at least one vacancy. Multiple, smaller-size vacancies, favored by statistics [10], are described by the increasing number of variables and their spectral signatures are less subjected, if at all, to motional narrowing. However, the isolated guest-vacancy pairs expectedly induce the larger dipoles (because of larger nearest asymmetry), so that the different vacancy contributions become balanced.

Shape analysis of $Q_{1}^{q}(0)$ line.-The $Q_{1}^{q}(0)$ shapes separated from the background were then fitted by the sum $a_{1} \sigma_{1}(z)+a_{2} \sigma_{2}(z)$, in which $\sigma_{1}(z)$ and $\sigma_{2}(z)$ represent the guest-vacancy and guest-guest contributions, respectively. The $\sigma_{1}(z)$ shape was derived from the $Q_{1}^{q}(0)$ profile at the lowest concentration ( $x=0.003$, Fig. 2$)$. It is well fitted by a weighted sum of two Lorentzians, modified for quantum asymmetry, and having HWHHs of 4.3 and $34.6 \mathrm{~cm}^{-1}$. The broader pedestal component bears about $80 \%$ of the $\sigma_{1}(z)$ intensity; the less intense narrow Lorentzian can be associated with the less probable larger vacancies. Finally, $\sigma_{2}(z)$ was taken to be a modified Lorentzian with variable HWHH $\left(\Gamma_{d}\right)$ and position of maximum $\left(\nu_{m}\right)$. The 4-parameter $\left(a_{1}, a_{2}, \Gamma_{d}, \nu_{m}\right)$ model excellently fits all (15) measured $Q_{1}^{q}(0)$ shapes (Fig. 3). The concentration behavior found, $a_{1} \sim x$ and $a_{2} \sim x^{2}$, agrees both with the assumed nature of the profiles and the integrated intensity behavior versus $x$. The guest-guest line is slightly shifted (see above) and broadened with $x: \Gamma_{d}\left(\mathrm{~cm}^{-1}\right)=1.73(1)+$ 5.2(5) $x$, due to vibrational dephasing. However, the latter effect is too weak to compete with the overall $Q_{1}^{q}(0)$ line narrowing caused by the growing guest-guest component (Fig. 2).

Diffusional narrowing of guest-guest lines.-Theoretically, the density transformation of the binary translational spectrum $\sigma_{2}(\Delta \omega)$ is characterized by the ratio $\chi$ of the lifetime $t_{c}$ of $\mathbf{m}^{\prime}$ to that $\left(t_{v}^{\prime}\right)$ of the relative translational velocity v. In a gas with isolated binary collisions $(x \ll 1)$, the shape is entirely defined by the intracollisional dynamics. The density growth shortens $t_{v}^{\prime}$ and starts to deform the shape at moderate values of $x$. In liquid, chaotic velocity variations become quasioscillatory that further shortens $t_{v}^{\prime}$ and finally causes motional narrowing at $x \gg 1$ : whatever 


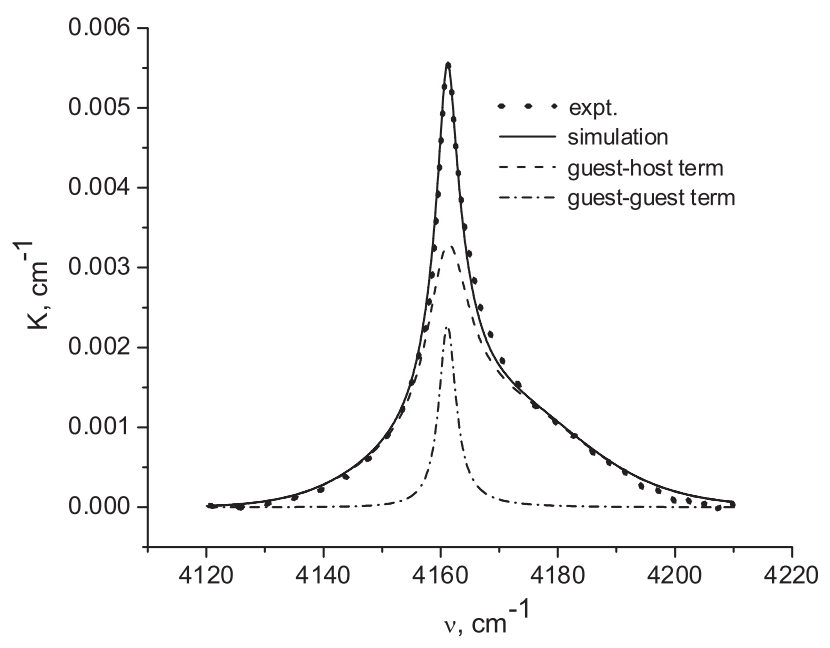

FIG. 3. Experimental and simulated $Q_{1}^{q}(0)$ shapes of $p-\mathrm{H}_{2}$ solution at $x=0.011, T=25.0 \mathrm{~K}$.

the spectrum was at $x \ll 1$, a sharper, quasi-Lorentzian line is formed instead. According to the general memory function approach [5], the appearance of a Lorentzian spectrum of a quantity $A$ suggests that the spectrum $\Gamma(\Delta \omega)$ of the time derivative $\dot{A}$ is converted by outer perturbations into a broad distribution. Moreover, the faster the variations of $\dot{A}$, the slower are those of $A$ whose spectrum becomes motionally narrowed and is said to be controlled by generalized diffusion [5,11]. In this regime, the Lorentzian HWHH $\Gamma$ equals $\Gamma(0)$. Applying this picture and treating the guest-guest term evolution classically, we conclude $\Gamma$ to be the integral over the time autocorrelation function of the dipole moment time derivative [5] $\dot{\mathbf{m}}^{\prime}=(\mathbf{v}, \boldsymbol{\nabla}) \mathbf{m}^{\prime}$. Since the translations are retarded $\left(t_{c} \gg\right.$ $\left.t_{v}^{\prime}\right)$, the time variation of the dipole moment gradient $\nabla \mathbf{m}^{\prime}$ in the integrand of $\Gamma$ can be disregarded and $\Gamma$ becomes proportional to $t_{v}^{\prime}$. Similar motional narrowing manifests itself in the binary guest-vacancy spectrum [sharper component of $\sigma_{1}(\Delta \omega)$ ], but, naturally, the vacancy signatures can be traced only in condensed matter. Notice also that the outlined theory of the newly found translational features closely resembles the motional narrowing of the spinrotation splitted NMR signals [12] and of the allowed rotational spectra [11] by angular-velocity relaxation but, to our knowledge, has never been properly applied to the induced spectra.

The motional narrowing regime $(x \gg 1)$ is independently supported by the data on cold neutron scattering by $\mathrm{H}_{2}$ in $\mathrm{LNe}$ [13], which result in $t_{v}^{-1} / 2 \pi c \approx 570 \mathrm{~cm}^{-1}$, and by the accurate quantum calculations [14] giving HWHH of the isotropic $Q_{1}(0)$ shape in pure gas to be about $100 \mathrm{~cm}^{-1}$; hence, $x \approx 6$. Actually, this quick estimation of $x$ seems to be much underrated because the used velocity correlation time $t_{v}$ does not contain the radial weighting function $\Phi(R)=\left(\nabla \mathbf{m}^{\prime}, \nabla \mathbf{m}^{\prime}\right)$ which enters $t_{v}^{\prime}$ relevant to our case. This weighting strongly favors the contribution from shorter distances with high accelerations whereas $t_{v}$ is contributed by softer interactions; this implies $t_{v}^{\prime}<t_{v}$.

Motional narrowing is also evidenced by four weak, guest-guest induced, double transitions $S_{1}(J)+S_{0}\left(J^{\prime}\right)$ (with $\left.J, J^{\prime}=0,1\right)$ which appear in the $\mathrm{H}_{2}$ spectra $(x \approx$ $0.06)$ as Lorentzians with $\Gamma \approx 5 \mathrm{~cm}^{-1}$. The $\sqrt{T}$ scaling of the HWHHs obtained for these transitions in the cooled gas ( $T=77 \mathrm{~K}[15])$ gives $\Gamma \approx 31 \mathrm{~cm}^{-1}$ that testifies to the pronounced narrowing at the gas-liquid transition.

Conclusion.-We have presented the first and direct spectroscopic evidences that the CIA lines due to the binary polarization, whatever they were in a gas, are transformed by diffusion into sharper Lorentzians in the liquid. Studies of the newly found features hold great promise for a new insight into the fluid translational microdynamics.

Useful discussions with M. O. Bulanin are appreciated. We thank the Special Research Fund of the University of Antwerp and the FWO-Vlaanderen for financial support.

*alex@ak1197.spb.edu

[1] G. E. Ewing and S. Trajmar, J. Chem. Phys. 41, 814 (1964).

[2] H. R. Zaidi and J. Van Kranendonk, Can. J. Phys. 49, 385 (1971).

[3] J. De Remigis, J. W. Mactaggart, and H. L. Welsh, Can. J. Phys. 49, 381 (1971).

[4] U. Buontempo et al., Can. J. Phys. 61, 156 (1983).

[5] D. Forster, Hydrodynamic Fluctuations, Broken Symmetry and Correlation Functions (Benjamin, Reading, MA, 1975).

[6] W. Herrebout, B. Van der Veken, A. P. Kouzov, and M. O. Bulanin, Phys. Rev. Lett. 92, 023002 (2004).

[7] J. Borysow, M. Moraldi, and L. Frommhold, Mol. Phys. 56, 913 (1985).

[8] A.P. Kouzov and V. A. Krasheninnikov, Opt. Spectrosc. 63, 154 (1987).

[9] W. K. Bischel and M. J. Dyer, Phys. Rev. A 33, 3113 (1986).

[10] B. Sadigh, M. Dzugutov, and S. R. Elliott, Phys. Rev. B 59, 1 (1999).

[11] A.I. Burshtein and S.I. Temkin, Spectroscopy of Molecular Rotation in Gases and Liquids (Cambridge University Press, Cambridge, England, 1994), Chap. 2.

[12] A. Abragam, The Principles of Nuclear Magnetism (Oxford University, Oxford, 1961).

[13] O. J. Eder, S. H. Chen, and P. A. Egelstaff, Proc. Phys. Soc. London 89, 833 (1966).

[14] W. Meyer, A. Borysow, and L. Frommhold, Phys. Rev. A 40, 6931 (1989).

[15] A. Sen, R. D. G. Prasad, and S. Paddy Reddy, J. Chem. Phys. 72, 1716 (1980). 